\title{
HH 222: A GIANT HERBIG-HARO FLOW FROM THE QUADRUPLE SYSTEM V380 ORI
}

\author{
Bo Reipurth ${ }^{1}$, John Bally ${ }^{2}$, Colin Aspin ${ }^{1}$, M. S. Connelley ${ }^{1}$, T. R. Geballe ${ }^{3}$, \\ Stefan Kraus ${ }^{4}$, Immo Appenzeller ${ }^{5}$, and Adam Burgasser ${ }^{6}$ \\ ${ }^{1}$ Institute for Astronomy, University of Hawaii at Manoa, 640 North Aohoku Place, Hilo, HI 96720, USA; \\ reipurth@ifa.hawaii.edu,caa@ifa.hawaii.edu,msc@ifa.hawaii.edu \\ ${ }^{2}$ Center for Astrophysics and Space Astronomy, University of Colorado, Boulder, CO 80309, USA; John.Bally@ colorado.edu \\ ${ }^{3}$ Gemini Observatory, 670 North Aohoku Place, Hilo, HI 96720, USA; tgeballe@ gemini.edu \\ ${ }^{4}$ Harvard-Smithsonian Center for Astrophysics, 60 Garden Street, MS-78, Cambridge, MA 02138, USA; stefan.kraus@cfa.harvard.edu \\ ${ }^{5}$ Landessternwarte Heidelberg, Königstuhl 12, D-69117 Heidelberg, Germany; iappenze@1sw.uni-heidelberg.de \\ ${ }^{6}$ Center for Astrophysics and Space Science, University of California San Diego, La Jolla, CA 92093, USA; aburgasser@ucsd.edu \\ Received 2013 March 1; accepted 2013 July 11; published 2013 October 7
}

\begin{abstract}
HH 222 is a giant shocked region in the L1641 cloud, and is popularly known as the Orion Streamers or "the waterfall" on account of its unusual structure. At the center of these streamers are two infrared sources coincident with a nonthermal radio jet aligned along the principal streamer. The unique morphology of $\mathrm{HH} 222 \mathrm{has}$ long been associated with this radio jet. However, new infrared images show that the two sources are distant elliptical galaxies, indicating that the radio jet is merely an improbable line-of-sight coincidence. Accurate proper motion measurements of HH 222 reveal that the shock structure is a giant bow shock moving directly away from the well-known, very young, Herbig Be star V380 Ori. The already known Herbig-Haro object HH 35 forms part of this flow. A new Herbig-Haro object, HH 1041, is found precisely in the opposite direction of $\mathrm{HH} 222$ and is likely to form part of a counterflow. The total projected extent of this HH complex is $5.3 \mathrm{pc}$, making it among the largest $\mathrm{HH}$ flows known. A second outflow episode from V380 Ori is identified as a pair of HH objects, HH 1031 to the northwest and the already known $\mathrm{HH} 130$ to the southeast, along an axis that deviates from that of $\mathrm{HH} 222 / \mathrm{HH}$ 1041 by only 3.7. V380 Ori is a hierarchical quadruple system, including a faint companion of spectral type M5 or M6, which at an age of $\sim 1$ Myr corresponds to an object straddling the stellar-to-brown dwarf boundary. We suggest that the HH 222 giant bow shock is a direct result of the dynamical interactions that led to the conversion from an initial non-hierarchical multiple system into a hierarchical configuration. This event occurred no more than 28,000 yr ago, as derived from the proper motions of the HH 222 giant bow shock.
\end{abstract}

Key words: Herbig-Haro objects - ISM: individual objects (HH 222) - ISM: jets and outflows - proper motions stars: individual (V380 Ori) - stars: pre-main sequence

Online-only material: color figures, supplemental data

\section{INTRODUCTION}

HH 222 (sometimes referred to as the Orion Streamers) is a very large and unique emission structure in the northern part of the L1641 cloud in Orion in the region of the $\mathrm{HH} 34$ and $\mathrm{HH}$ $1 / 2$ flows. Its existence was apparently first commented upon in the literature by Cohen \& Schwartz (1983).

Interference filter images and spectra have demonstrated that HH 222 is a large emission line structure. The HH 222 core itself has many similarities to the many other Herbig-Haro $(\mathrm{HH})$ flows in the region and is located in the vicinity of young stars, hence its inclusion among the HH objects (Reipurth 1999). What makes it unique and makes it stand out from all other known $\mathrm{HH}$ objects is a couple of very large streamers, the longest of which stretches across 260 arcsec, corresponding to $0.6 \mathrm{pc}$ at the often-assumed distance of $460 \mathrm{pc}$ for the Orion clouds (see Section 3.1). Reipurth \& Sandell (1985) found HH 222 to be situated near the edge of a molecular cloud, with the T Tauri star V571 Orionis located only 15 arcsec from the center of the shock structure, and they suggested that HH 222 could be the result of a T Tauri wind impacting the cloud edge. This idea was abandoned when Yusef-Zadeh et al. (1990) detected a strong radio continuum jet at the core of the shock structure, with a large-scale non-thermal radio flow pointing along the longest of the streamers. They also identified two faint near-infrared sources around the location of the radio continuum source. The
Very Large Array (VLA) source was also detected by Morgan et al. (1990). The source was not detected in the $1300 \mu \mathrm{m}$ survey of HH energy sources by Reipurth et al. (1993). Most recently, Trejo \& Rodríguez (2010) have presented new highresolution $6 \mathrm{~cm}$ and $20 \mathrm{~cm}$ radio continuum maps of the radio continuum jet.

Non-thermal radio sources in low mass star forming regions are rare, the best known case is the triple source in Serpens, which at high resolution has been revealed to be a fine bipolar radio jet (Rodríguez et al. 1989; Curiel et al. 1993; Raga et al. 2000). The presence of non-thermal emission in such regions is not very easy to explain, but may involve diffusive shock acceleration of particles (Crusius-Wätzel 1990; Henriksen et al. 1991).

Castets et al. (2004) mapped the region around HH 222 in several millimeter transitions, but did not detect any dense molecular cloud core that could harbor a young driving source. Among several alternative explanations for a driving source, they considered the possibility of an evolved source accidentally passing through the Orion clouds.

In this paper, we present optical and infrared observations of the HH 222 complex, measure proper motions of the streamers, and find that $\mathrm{HH} 222$ is not driven by an internal source, but is a giant bow shock driven by the Herbig Be star V380 Ori located 22 arcmin to the southeast. We argue that the two infrared sources at the center of $\mathrm{HH} 222$ are distant elliptical galaxies, 
and conclude that the associated radio continuum jet at the center is an extragalactic object.

\section{OBSERVATIONS}

The first-epoch CCD images were obtained on 1994 January 13 with the ESO $3.5 \mathrm{~m}$ New Technology Telescope (NTT) at La Silla. EMMI was used with a Loral CCD providing a field of view of about $9 \times 10$ arcmin at a scale of 0.35 arcsec per pixel. The seeing was approximately 0.7 arcsec. Three exposures through both $\mathrm{H} \alpha$ and [S II] narrowband filters were obtained for a total exposure time of $2700 \mathrm{~s}$ per filter.

The second-epoch CCD images were made with the $8 \mathrm{~m}$ Subaru telescope and SuprimeCam. A five-point dither map with step size of $40^{\prime \prime}$ was used in order to cover the $17^{\prime \prime}$ gaps between the 10 individual CCDs. An $\mathrm{H} \alpha$ filter was used with an FWHM of $99 \AA$, central wavelength of $6596 \AA$, and peak transmission of $87 \%$. The $\mathrm{H} \alpha$ image was obtained in clear conditions on 2006 January 4 with a total exposure time of $5 \times 12$ minutes at airmass 1.15 , resulting in images with FWHM of 0.9 . A [S II] filter was used with an FWHM of $130 \AA$, central wavelength of $6714 \AA$, and peak transmission of $87 \%$. The [S II] image was obtained in clear conditions on 2006 January 5 with a total exposure time of $5 \times 12$ minutes at airmass 1.22 , and the seeing varied from 0 .'61 to 0.65 between exposures.

Infrared images through $J, H, K s$, and $\mathrm{H}_{2}$ filters were obtained of the HH 222 core region using NIRI on the Gemini-North telescope on 2006 October 14. The broadband images were each exposed for $10 \times 60 \mathrm{~s}$ on-source, while the $\mathrm{H}_{2}$ images were exposed for $10 \times 2$ minutes on-source. The photometry was calibrated using the standard FS13. Near-infrared longslit spectroscopy with both IRS 1 and IRS 2 under the slit was obtained on 2006 November 13 also using the GeminiNorth telescope and NIRI and the $K$-band grism. The F6 camera was used with a 4 pixel wide slit, and the spatial scale was 0 ". 12 pixel $^{-1}$. The total on-source integration time was $40 \times$ 2 minutes. Telluric lines were removed by observing the A0 standard HIP 23661.

HH 222 was observed with the echelle spectrograph at the $3.5 \mathrm{~m}$ Apache Point Observatory on the nights of 2002 November 21 and 22. This cross-dispersed spectrograph has a resolution of about 32,000 and around the $\mathrm{H} \alpha$ and [S II] doublet has a dispersion of $0.0814 \mathrm{~A} \mathrm{pixel}^{-1}\left(3.69 \mathrm{~km} \mathrm{~s}^{-1}\right.$ pixel $\left.^{-1}\right)$ and $0.08027 \mathrm{~A} \mathrm{pixel}^{-1}\left(3.72 \mathrm{~km} \mathrm{~s}^{-1}\right.$ pixel $\left.^{-1}\right)$ respectively. The brightest knot in the [S II] image located at $J(2000)=$ 05:35:42.0, - 06:23:01 was targeted with a 1.5 arcsec by 3 arcsec rectangular slit. A position 30 arcsec due north of this location was observed to obtain a sky reference spectrum. Three $180 \mathrm{~s}$ duration exposures were obtained at each location, median combined, and the resulting cosmic-ray-free spectra were differenced. This procedure provides a direct measurement of the line-width and line center velocity of the emission from $\mathrm{HH} 222$ relative to the diffuse nebular emission that permeates the entire field of view surrounding $\mathrm{HH} 222$. All radial velocity and line-width measurements are referenced to the central velocity of the $\mathrm{H} \alpha$ emission line associated with the diffuse nebular emission.

A low dispersion, long slit optical spectrum of the core of HH 222 was recorded on 2000 February 3 during the commissioning of the FORS2 instrument at the $8.2 \mathrm{~m}$ unit telescope 2 of ESO's Very Large Telescope (VLT). The 418 arcsec slit was oriented at position angle (P.A.) $35^{\circ}$ along the longest and brightest streamer of the HH 222 complex. Using Grism 300V and a slit width of 1 arcsec, the spectrum provides a resolution of $0.235 \mathrm{~nm}$ and was integrated for $1800 \mathrm{~s}$ on source. For data reduction, we used the ESO FORS2 data reduction pipeline (release 4.8.7), which performs a bias and flatfield correction, dispersion correction, and wavelength-calibration. In order to separate the background emission from HH 222, the highly irregular sky background around $\mathrm{HH} 222$ was estimated from our CCD images, and a region along the slit, $\sim 40$ arcsec further northeast, was chosen to correct for the sky.

A medium resolution $(R \sim 750) 0.9-2.4 \mu \mathrm{m}$ spectrum was obtained of V380 Ori B on 2012 December 28 using SpeX in the short cross-dispersed mode on the $3.0 \mathrm{~m}$ NASA Infrared Telescope Facility at Mauna Kea (Rayner et al. 2003). The $0 \prime .8 \times 15^{\prime \prime}$ long slit was placed perpendicular to the direction between V380 Ori A and B. Total exposure time was 40 minutes. Subsequently $J H K$ images were obtained in photometric conditions and with a seeing of $0{ }^{\prime} 8$.

A medium resolution $(R \sim 1800) 1-2.5 \mu \mathrm{m}$ spectrum of V380 Ori B was obtained on 2013 January 16 at the Gemini-North telescope using the facility instrument GNIRS in its cross-dispersed mode. A 0.3 arcsec wide slit was employed and oriented at P.A. $30^{\circ}$ in order to minimize contamination from the much brighter A component of the system. The spectrum was obtained in clear skies and $1^{\prime \prime}$ seeing, utilizing the standard stare/nod-along-slit mode, with a beam separation of $4^{\prime \prime}$. The total exposure time was 40 minutes. A nearby A0 star was used as a flux and telluric standard. Due to variable seeing conditions and the narrow slit, the flux calibration is uncertain by a factor of $\sim 1.5$.

\section{RESULTS}

\subsection{Optical Imaging and Proper Motions}

In Figure 1 we show the deepest image of HH 222 taken so far, using SuprimeCam on the $8 \mathrm{~m}$ Subaru telescope. The figure is a combination of a $1 \mathrm{hr}$ exposure through an $\mathrm{H} \alpha$ filter and another $1 \mathrm{hr}$ exposure through a [S II] filter, both taken in sub-arcsecond seeing (see Section 2 for details). HH 222 has a bright core, from which several streamers emanate, giving it its characteristic appearance, unique among $\mathrm{HH}$ objects. It is noticeable that many structures have edges that are sharper toward the NNW. The total extent of the object exceeds 6 arcmin.

The distance to HH 222 is uncertain. Recently, Menten et al. (2007) derived a distance of 414 pc for M42, just north of $\mathrm{HH}$ 222. Wilson et al. (2005) suggests a distance of $465 \mathrm{pc}$ for the central part of the cloud complex that contains $\mathrm{HH} 222$, consistent with the value of $460 \mathrm{pc}$ that has been used frequently in the past for L1641, and which we continue to use in this paper. At a distance of $460 \mathrm{pc}$, the 6 arcmin extent of $\mathrm{HH} 222$ corresponds to a projected dimension of 165,000 AU, or $\sim 0.8 \mathrm{pc}$.

We have also subtracted the $\mathrm{H} \alpha$ and [S II] images, and the result is shown in Figure 2. $\mathrm{H} \alpha$-strong regions are black, and dominate the region. [S II]-strong features are white, and the main such structure (in the following dubbed "the [S II]-knot") is seen just southeast of the main core. Other faint [S II]-strong features are similarly preferably shifted to the southeast, and we discuss this further below.

We have derived proper motions for various components of HH 222 using the abovementioned Subaru images combined with similar images obtained at the $3.5 \mathrm{~m}$ ESO NTT in 1994 (see Section 2), providing a time span of 4375 days, or $12 \mathrm{yr}$. 


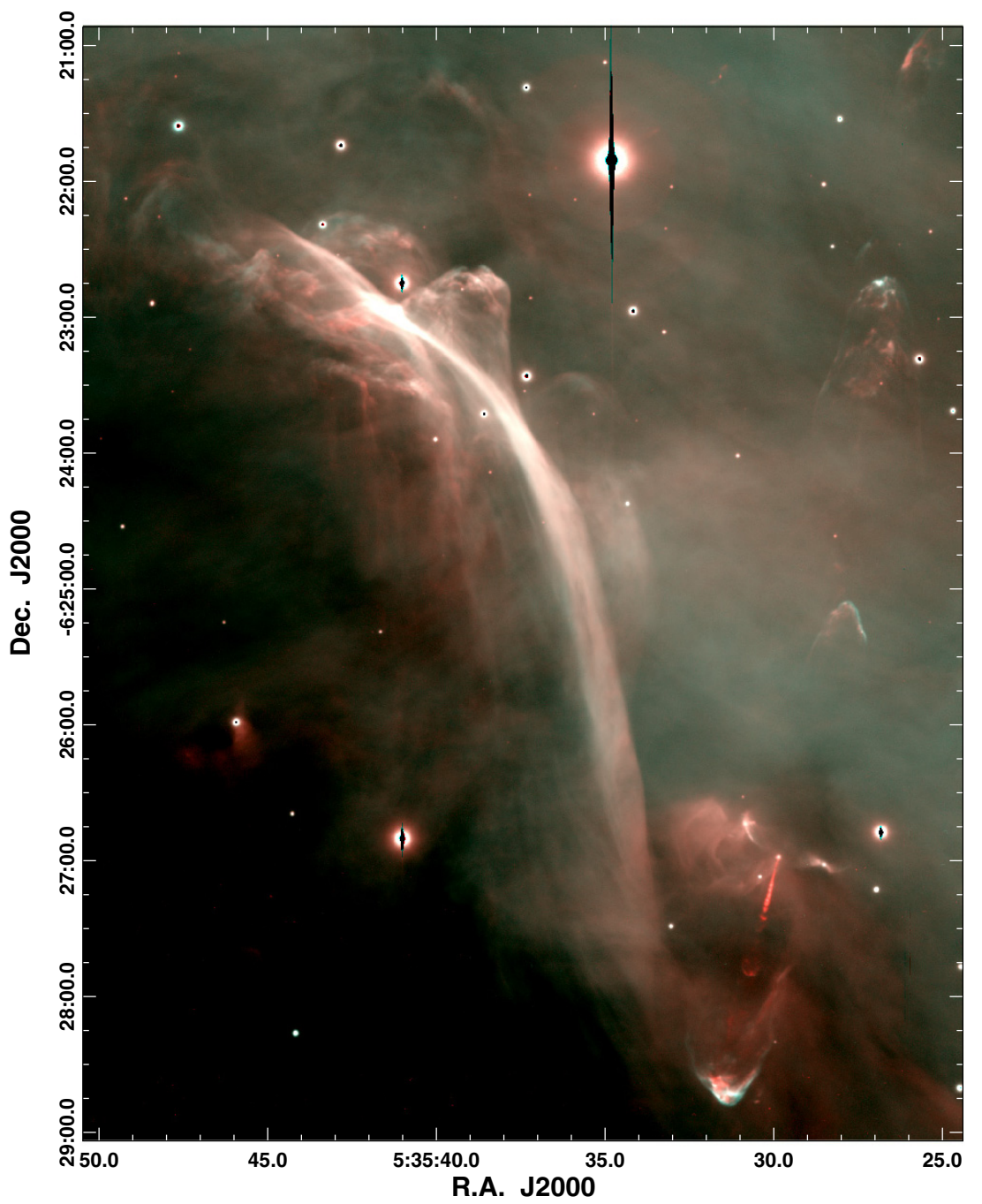

Figure 1. Combined $\mathrm{H} \alpha$ and [S II] image of the HH 222 region, taken at the $8 \mathrm{~m}$ Subaru telescope with SuprimeCam, with parts of the HH 34 giant flow complex to the west. Shock regions that are strong in [S II] are reddish. North is up and east is to the left.

(A color version of this figure is available in the online journal.)

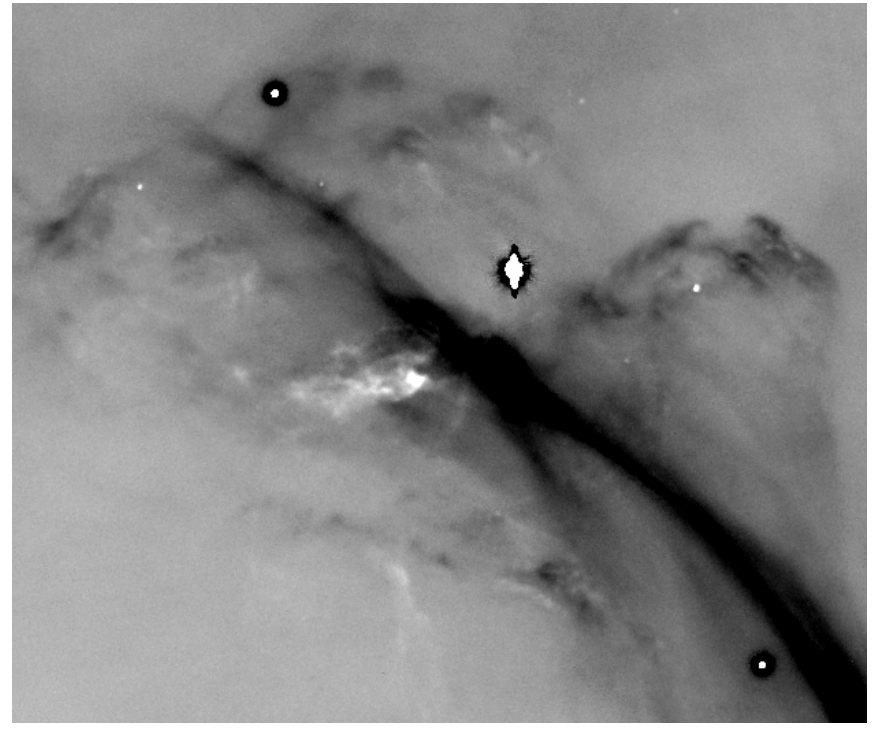

Figure 2. $\mathrm{H} \alpha-[\mathrm{S} \mathrm{II}]$ difference image showing the central region of $\mathrm{HH} 222$ $\mathrm{H} \alpha$-strong regions are black, and $\left[\mathrm{S}_{\mathrm{II}}\right]$-strong regions are white. The figure is 2 arcmin wide, corresponding to $0.28 \mathrm{pc}$ at the assumed distance of $460 \mathrm{pc}$.
Neither our IRAF-based nor IDL-based automated proper motion scripts, which we usually use for determination of tangential velocities of $\mathrm{HH}$ objects, worked well for $\mathrm{HH} 222$ because it is so diffuse: slight intensity variations along the $\mathrm{HH}$ 222 shocks tended to result in gross transverse motions. Instead we used a more manual method as described below. The NTT and Subaru images were cropped, re-sampled to a common area and pixel scale, and scaled to match in intensity. The 1994 NTT image was registered to the 2006 Subaru image with IRAF tasks GEOMAP and GEOTRAN to remove geometric distortions using 15 stars that were visible in both images, but not saturated in either.

Sharp-edged and compact structures were marked on the 2006 $\mathrm{H} \alpha$ image using a DS9 REGION file. This file was copied onto the $1994 \mathrm{H} \alpha$ image, and the centroids of each region were moved to provide the best fit to each marked feature as judged by eye. An IDL routine was used to determine the difference in the centroid positions of regions on the 1994 and 2006 images, to compute the proper motion vectors, and to generate a REGION file of these vectors which was then overlaid on the 2006 Subaru $\mathrm{H} \alpha$ image to produce the figure shown in Figure 3; the vector lengths correspond to the motion expected in $300 \mathrm{yr}$. The mean proper motion of the entire $\mathrm{HH} 222$ complex was measured to 


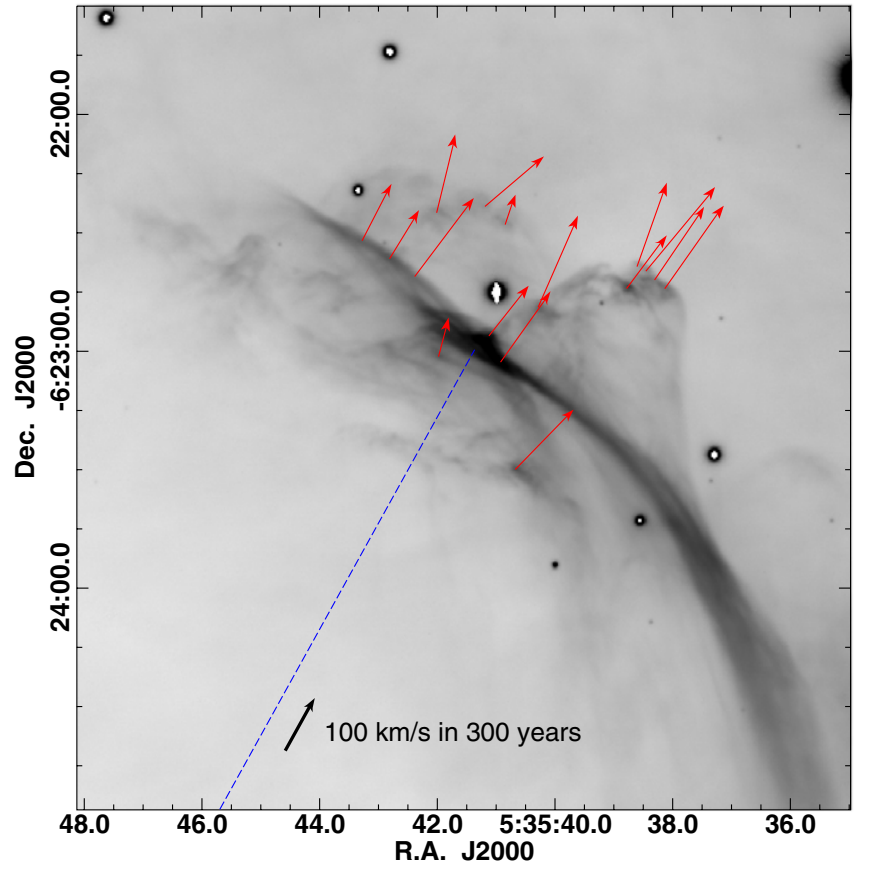

Figure 3. Proper motions of $\mathrm{HH} 222$. Tangential motions of individual features are indicated by red arrows, which vary around a mean of $107 \pm 25 \mathrm{~km} \mathrm{~s}^{-1}$; arrows show the expected motion in $300 \mathrm{yr}$. The black arrow shows the physical displacement that would take place in $300 \mathrm{yr}$ assuming a motion of $100 \mathrm{~km} \mathrm{~s}^{-1}$. The dashed blue line indicates the direction to V380 Ori.

(A color version of this figure is available in the online journal.)

$V_{\mathrm{pm}}=107 \pm 25 \mathrm{~km} \mathrm{~s}^{-1}$ toward P.A. $=329^{\circ} \pm 10^{\circ}$ (random error).

We note the following:

1. The proper motion of the HH 222 complex is perpendicular to the main set of streamers. It is also consistent with the axes of symmetry of the three or four bow shaped shocks that protrude ahead of the main HH 222 streamers. These facts provide strong evidence that $\mathrm{HH} 222$ is a giant working surface moving through the L1641 star forming region.
2. The $\mathrm{H} \alpha$-[S II] difference image separates stronger from weaker shocks, and we see that the main core of HH 222 is located ahead of the [S II]-bright region, in the direction of motion. This is consistent with the expectation that a working surface consists of a bow shock, where the ambient medium is shocked and accelerated, and a Mach disk where jet material is shocked and decelerated. The fact that the Mach disk is a weaker shock indicates that the jet is heavy relative to the ambient medium.

3. The direction of the tangential motion of $\mathrm{HH} 222$ points directly away from the Herbig Be star V380 Ori, one of the youngest Herbig Ae/Be stars known, still associated with the primordial material from which it was born (Herbig 1960). While the mean HH 222 proper motion vector is at P.A. $=329^{\circ} \pm 10^{\circ}$, the direction from V380 Ori to the core of $\mathrm{HH} 222$ is $331^{\circ}$ (indicated by the dotted line in Figure 3 ).

4. The projected extent of the HH 222 outflow lobe from V380 Ori is $3.0 \mathrm{pc}$. If the current tangential motion of $\mathrm{HH} 222$ has been constant, the age of HH 222 is 28,000 yr. However, it is likely that the velocity of the bow shock has declined with time (see Devine et al. 1997), making this an upper limit to the age of the shock structure.

\subsection{Optical Spectroscopy}

The emission lines in our low-dispersion spectrum of HH 222 (Figure 4) match very well the spectra of other HH objects, such as HH 1 (e.g., Solf et al. 1988). This supports our interpretation that, despite its unusual morphology, $\mathrm{HH} 222$ is a typical $\mathrm{HH}$ object. The identified lines and their relative line strengths are listed in Table 1. The overall appearance of the spectrum is that of a moderately excited $\mathrm{HH}$ object.

Using our high-resolution spectra, we find that the [S II] knot in HH 222 (prominently visible behind the main shock in Figure 2) exhibits a slight blueshift with respect to the diffuse nebular emission and has a significantly larger line-width in [S II]. The $\mathrm{H} \alpha$ emission line peak in the $\mathrm{HH} 222$ [S II] knot has a relative radial velocity of $V_{\text {rel }}=-6 \pm 10 \mathrm{~km} \mathrm{~s}^{-1}$, consistent with no Doppler shift, and a line width $\Delta V=$ $37 \pm 14 \mathrm{~km} \mathrm{~s}^{-1}$, about twice the width of the background diffuse emission. The brighter $\lambda 6717$ [S II] line is blueshifted

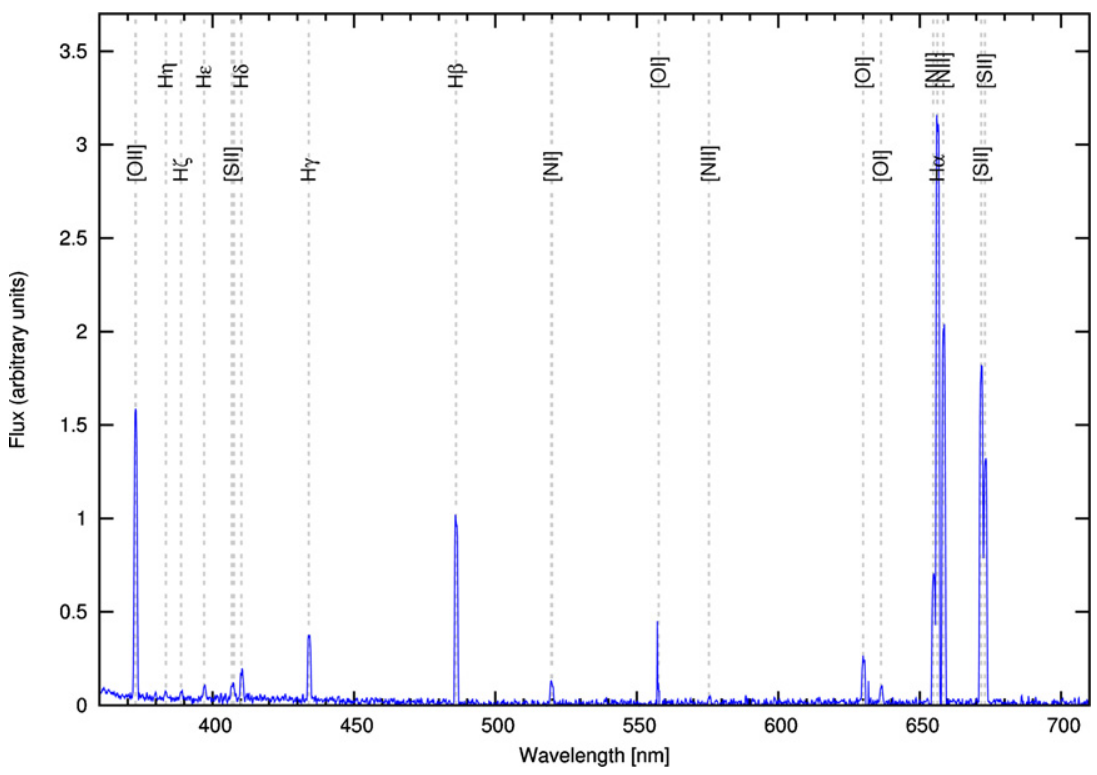

Figure 4. Spectrum of the core of HH 222 obtained with FORS2 on the VLT.

(A color version and supplemental data for this figure are available in the online journal.) 
Table 1

Lines Identified in the FORS2 Spectrum of $\mathrm{HH} 222$

\begin{tabular}{lcc}
\hline \hline $\begin{array}{l}\text { Wavelength } \\
(\mathrm{nm})\end{array}$ & Identification & $\begin{array}{c}\text { Relative Line Intensity } \\
(\mathrm{H} \beta=100)\end{array}$ \\
\hline 372.7 & {$\left[\mathrm{O}_{\mathrm{II}}\right]$} & 152 \\
383.5 & $\mathrm{H} \eta$ & 2.4 \\
388.9 & $\mathrm{H} \zeta$ & 3.1 \\
397.0 & $\mathrm{H} \epsilon$ & 6.6 \\
406.8 & {$[\mathrm{~S} \mathrm{II}]$} & 10 \\
410.2 & $\mathrm{H} \delta$ & 16 \\
434.1 & $\mathrm{H} \gamma$ & 34 \\
486.1 & $\mathrm{H} \beta$ & 100 \\
519.8 & {$[\mathrm{~N} \mathrm{I}]$} & 12 \\
557.7 & {$[\mathrm{O} \mathrm{I}]:$} & 12 \\
575.5 & {$[\mathrm{~N} \mathrm{II}]$} & 3.0 \\
630.0 & {$[\mathrm{O} \mathrm{I}]$} & 23 \\
636.3 & {$[\mathrm{O} \mathrm{I}]$} & 8.4 \\
654.8 & {$[\mathrm{~N} \mathrm{II}]$} & 68 \\
656.3 & $\mathrm{H} \alpha$ & 354 \\
658.3 & {$[\mathrm{~N} \mathrm{II}]$} & 217 \\
671.7 & {$[\mathrm{~S} \mathrm{II}]_{673.1}^{\left[\mathrm{S}_{\mathrm{II}}\right]}$} & 195 \\
& & 140 \\
& &
\end{tabular}

Note. The [O I] 557.7 line might contain contributions from nightglow and from the ambient nebula.

Table 2

Coordinates and Photometry of IRS 1 and 2

\begin{tabular}{lccccc}
\hline \hline Source & $\alpha_{2000}$ & $\delta_{2000}$ & $J$ & $H$ & $K s$ \\
\hline IRS 1 & $05: 35: 41.97$ & $-06: 23: 02.6$ & 18.62 & 17.02 & 15.93 \\
IRS 2 & $05: 35: 41.53$ & $-06: 23: 07.3$ & 19.51 & 17.92 & 16.73 \\
\hline
\end{tabular}

to $V_{\text {rel }}=-18 \pm 10 \mathrm{~km} \mathrm{~s}^{-1}$ and has a line width of $\Delta V=$ $48 \pm 14 \mathrm{~km} \mathrm{~s}^{-1}$. The fainter $\lambda 6731$ [S II] line is blueshifted to $V_{\text {rel }}=-30 \pm 10 \mathrm{~km} \mathrm{~s}^{-1}$, but the signal-to-noise is too low to make an accurate line-width measurement. The intensity ratio of the $[\mathrm{S}$ II] doublet is consistent with a flux ratio $I_{6717} / I_{6731}=1.4$, indicating that the plasma is in the lowdensity limit with $n<100 \mathrm{~cm}^{-3}$. These parameters, combined with the relatively bright emission in $\mathrm{H} \alpha$, imply that the $\mathrm{HH} 222$ recombination lines are formed in a limb-brightened sheet seen edge-on with a much greater extent along the lineof-sight than on the plane-of-the sky. This suggests that $\mathrm{HH}$ 222 moves close to the plane of the sky. We can, in fact, determine the angle of the HH 222 flow to the plane of the sky by using the radial and tangential velocities we have measured. If we adopt a weighted mean of the radial velocities for the [S II] 6717/6731 lines of $-22 \pm 10 \mathrm{~km} \mathrm{~s}^{-1}$ and a mean tangential velocity for $\mathrm{HH} 222$ of $107 \pm 25 \mathrm{~km} \mathrm{~s}^{-1}$, we conclude that HH 222 is indeed moving close to the plane of the sky, at an angle of only $\sim 12^{\circ} \pm 8^{\circ}$.

\subsection{Near-infrared Imaging and Spectroscopy}

The very core of $\mathrm{HH} 222$, from which all the streamers appear to emanate, is also the location of two faint near-infrared sources first noted by Yusef-Zadeh et al. (1990). Our infrared images obtained at the Gemini-North telescope (see Section 2) provide the coordinates and photometry listed in Table 2 . Additionally we have obtained low-dispersion $K$-band spectra of these two infrared sources; although the signal-to-noise was not sufficient to detect any absorption lines in the continuum, it is evident that the objects show no emission lines in the $K$ band. We have

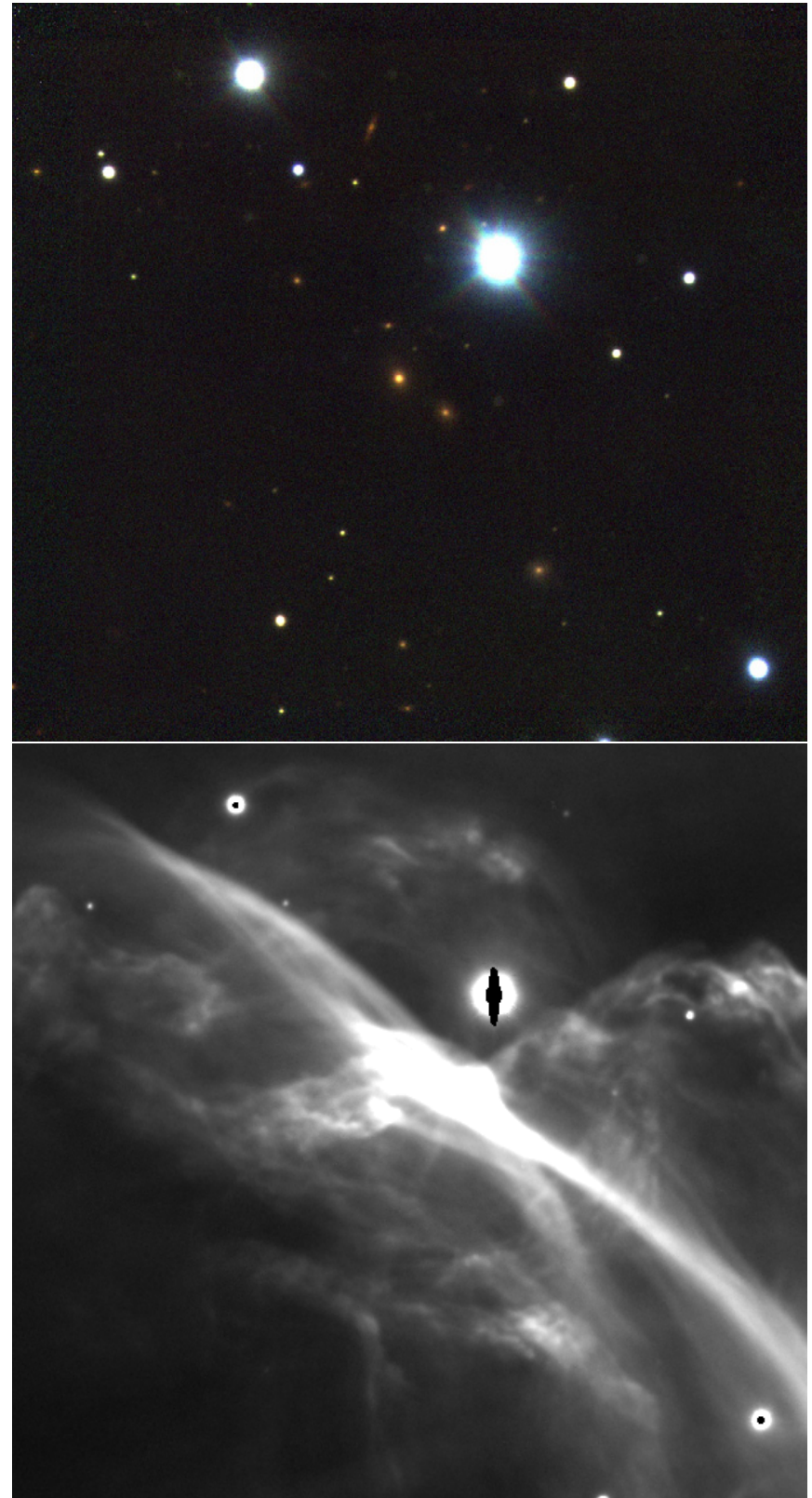

Figure 5. Infrared $J H K$ color mosaic of the central HH 222 region from the Gemini-North telescope (top) and a [S II] image of the same area from the Subaru telescope (bottom). The two infrared sources IRS 1 and IRS 2 are in the very center of the infrared image. The images are about 110 arcsec wide. North is up and east is to the left.

(A color version of this figure is available in the online journal.)

also examined the region using Spitzer IRAC data, and found no objects at the position of the two infrared sources, indicating that they are unlikely to be young stars. The near-infrared $J H K$ images are combined into a color figure shown in the upper panel of Figure 5, and to put the region in proper context the very same field is shown in the lower panel in the optical ([S II]). The infrared image shows that the two infrared sources at the core of the streamers are slightly extended and slightly elongated. In total, at least five other slightly nebulous objects, including two spiral galaxies, surround the two infrared sources. The appearance is that of a distant small cluster of galaxies with two spiral galaxies and at least two elliptical galaxies. 


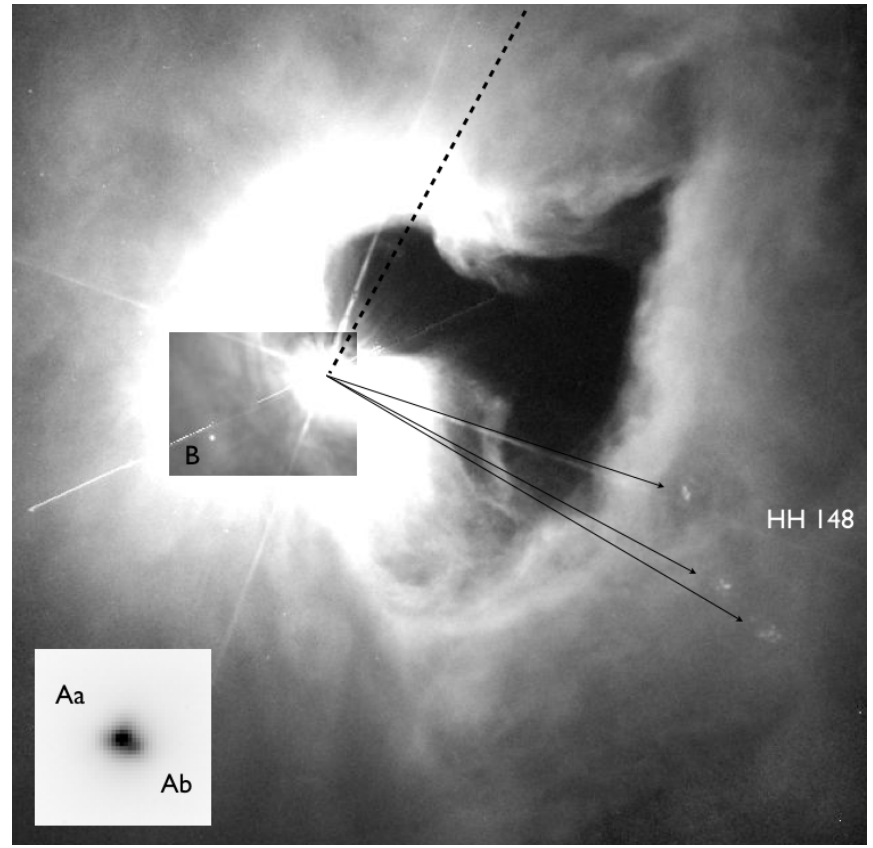

Figure 6. Archival HST image of the immediate surroundings of V380 Ori (Program 8548, PI: K. S. Noll). The dark structure is not a dense globule, but a cavity blown in the surrounding cloud. The image is taken with the filter F675W, which transmits the [S II] 6717/6731 lines, and shows three knots in $\mathrm{HH}$ 148. Directions from V380 Ori are indicated by arrows. The direction to $\mathrm{HH} 222$ is indicated by the dashed line. The image is approximately 1 arcmin across, corresponding to about 25,000 AU, with north up and east left. The inner over-exposed region around V380 Ori is shown with another cut, revealing star $\mathrm{B}$ at a distance of $8.8 \mathrm{arcsec}$ and P.A. $=120.4$ from V380 Ori. The inset at the lower left shows an archival $K$-band image of V380 Ori obtained with NACO at the ESO VLT, revealing the two components of V380 Ori (PIs: M. Petr-Gotzens and G. Dûchene).

\section{DISCUSSION}

A wide range of explanations for the origin of the curious "Orion Streamers" have been put forward over the past $25 \mathrm{yr}$ (e.g., Reipurth \& Sandell 1985; Yusef-Zadeh et al. 1990; Castets et al. 2004), all of which have been unsatisfactory. Our proper motion measurements of $\mathrm{HH} 222$ have shown a clear motion directly away from V380 Ori. This is the key to understand the nature of $\mathrm{HH} 222$, and here we summarize the basic features of V380 Ori.

\subsection{Properties of V380 Ori}

The rich emission line spectrum of V380 Ori (BD $-06^{\circ} 1253=$ Parenago $2393=$ HBC $164=$ Haro 4-235) was first noted by Herbig (1946) and Morgan \& Sharpless (1946). It was subsequently recognized as belonging to the class of Herbig Ae/Be stars (Herbig 1960), with a spectral type around B9 and a luminosity of $\sim 100 L_{\odot}$. V380 Ori has been observed in great detail and over many wavelength ranges (e.g., Herbig 1960; Hamann \& Persson 1992; Rossi et al. 1999; Stelzer et al. 2006). It is surrounded by a bright reflection nebula known as NGC 1999, which has been studied by Warren-Smith (1983) and Cardelli \& Böhm (1984). Close to V380 Ori is a dark protrusion discussed by Herbig (1946); CCD images obtained with the Hubble Space Telescope (HST) clearly show that this is not a dense dark cloud core, but a cavity in the bright reflection nebula (Figure 6), as discussed by Stanke et al. (2010).

V380 Ori is the most massive star in this part of the L1641 cloud, and the deep $\mathrm{H} \alpha$ image in Figure 14 shows that it illuminates a wide surrounding area; radial lines in the cloud

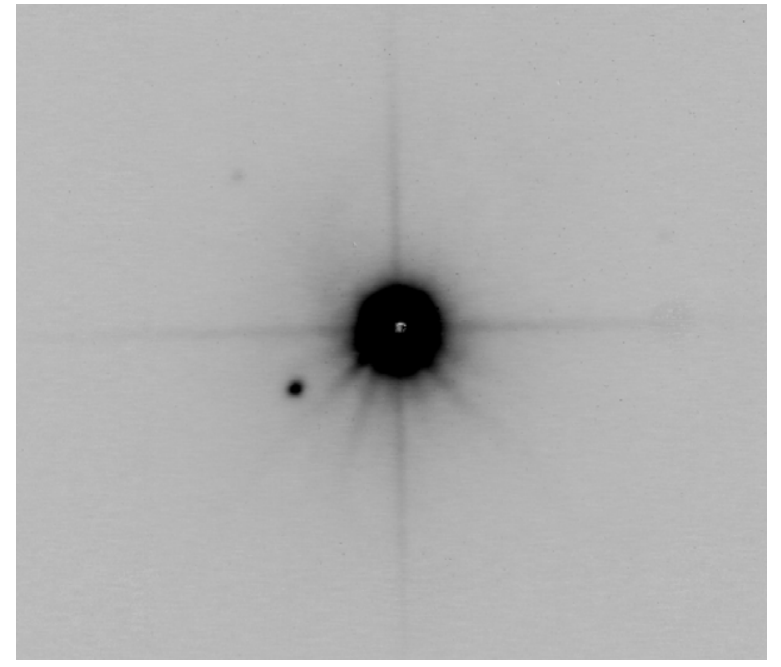

Figure 7. $K$-band image from the IRTF showing V380 Ori with its companion $\mathrm{B}$ at a separation of $9^{\prime \prime}$. The image is 1 arcmin across with north up and east to the left.

surface suggests a streaming motion centered on V380 Ori (Figure 12). Heyer et al. (1992) and Nakamura et al. (2012) found several partial ring like structures in ${ }^{13} \mathrm{CO}$ and ${ }^{12} \mathrm{CO}$ maps, respectively, which may be expanding shells.

Millan-Gabet et al. (2001) has used long baseline interferometry to detect and characterize the circumstellar disk around V380 Ori. They find an elongated structure with a good fit to the visibility data for a P.A. of $\sim 30^{\circ}$ (disk major axis, from north through east) and an inclination (of disk plane to plane of the sky) of $80^{\circ}$. In other words, the disk lies almost edge-on.

\subsection{V380 Ori as a Quadruple System}

V380 Ori has a close infrared companion, with a separation of $0^{\prime \prime} .15$ at P.A. $204^{\circ}$ (Leinert et al. 1997). The two components (labeled $\mathrm{Aa}$ and $\mathrm{Ab}$ ) can be seen in the archival NACO image from the ESO VLT (inserted in Figure 6; PIs: M. Petr-Gotzens and G. Dûchene). Possible evidence for orbital motion was detected by Smith et al. (2005), and is further corroborated in Figure 6. More recently, Alecian et al. (2009) found that V380 Ori Aa is itself a spectroscopic binary with a period of $\sim 104$ days, with the secondary being a massive $\mathrm{T}$ Tauri star. They determine an effective temperature for the primary of $10,500 \mathrm{~K}$, and for the secondary of $5500 \mathrm{~K}$, with a mass for V380 Ori of roughly $2.8 M_{\odot}$. They further find that a disk may surround this binary and that a wind emanates from the system. Altogether, V380 Ori is a hierarchical triple system.

We have found evidence for yet another component in this multiple system. When examining the optical HST image in Figure 6 we noted a faint star at a distance of $8^{\prime \prime} .8$ at P.A. 120.4. Examining the $K^{\prime}$-band atlas of Hodapp (1994) we found the source to be quite bright at $2.2 \mu \mathrm{m}$. The only information we have found in the literature on this star is by Corcoran \& Ray (1995), who detected it at $K \sim 13.0$ and called it V380 Ori B, a label we retain here. In Figure 7 we show a $K$-band image obtained at the IRTF; photometry yields $J=14.059 \pm 0.010$, $H=13.267 \pm 0.008$, and $K=12.772 \pm 0.026$. In Figure 8 we show a 1.0-2.5 $\mu \mathrm{m}$ spectrum of this component. The abundant presence of $\mathrm{H}_{2} \mathrm{O}$ and $\mathrm{CO}$ bands together with lines of $\mathrm{Na}, \mathrm{K}$, $\mathrm{Ca}$, etc, indicate a mid-to-late $\mathrm{M}$ spectral type. Figure 9 shows a series of best $\chi^{2}$ fits to late spectral standards with interstellar extinction as a free parameter using the Cardelli et al. (1989) 


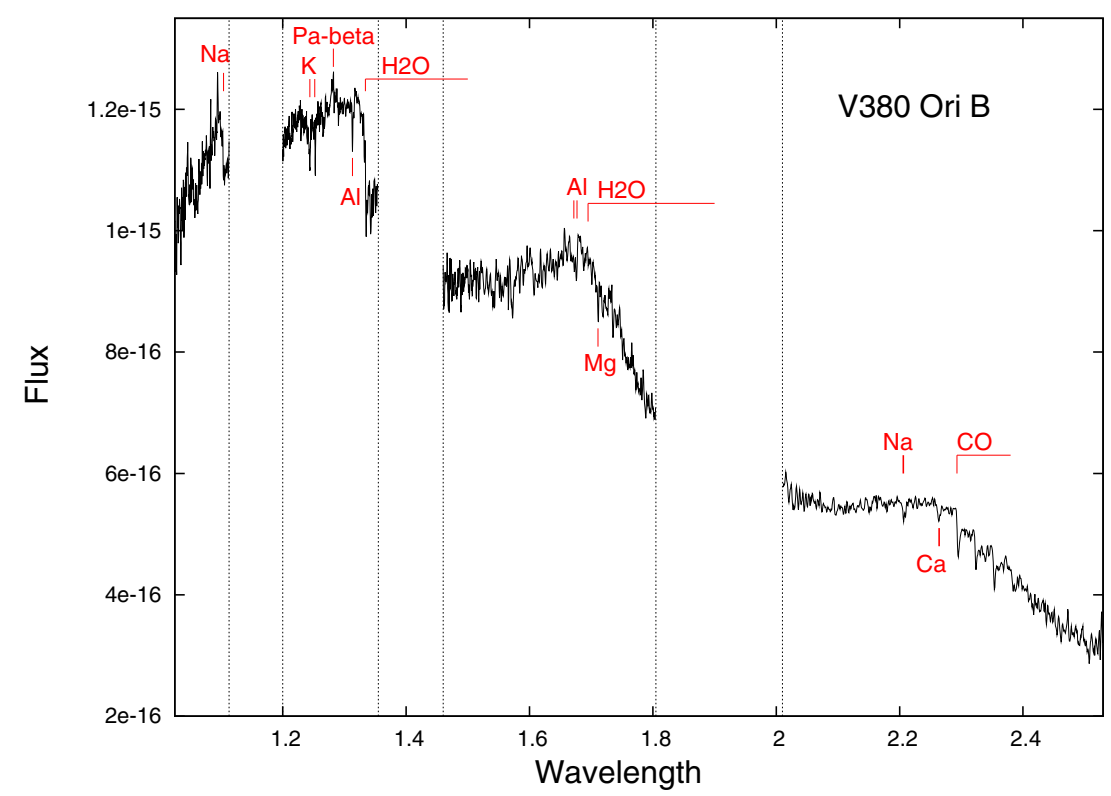

Figure 8. Near-infrared GNIRS spectrum of the faint companion V380 Ori B reveals that it is a borderline brown dwarf of spectral type M5-M6. Three noisy sections with poor atmospheric transmission are marked.

(A color version of this figure is available in the online journal.)

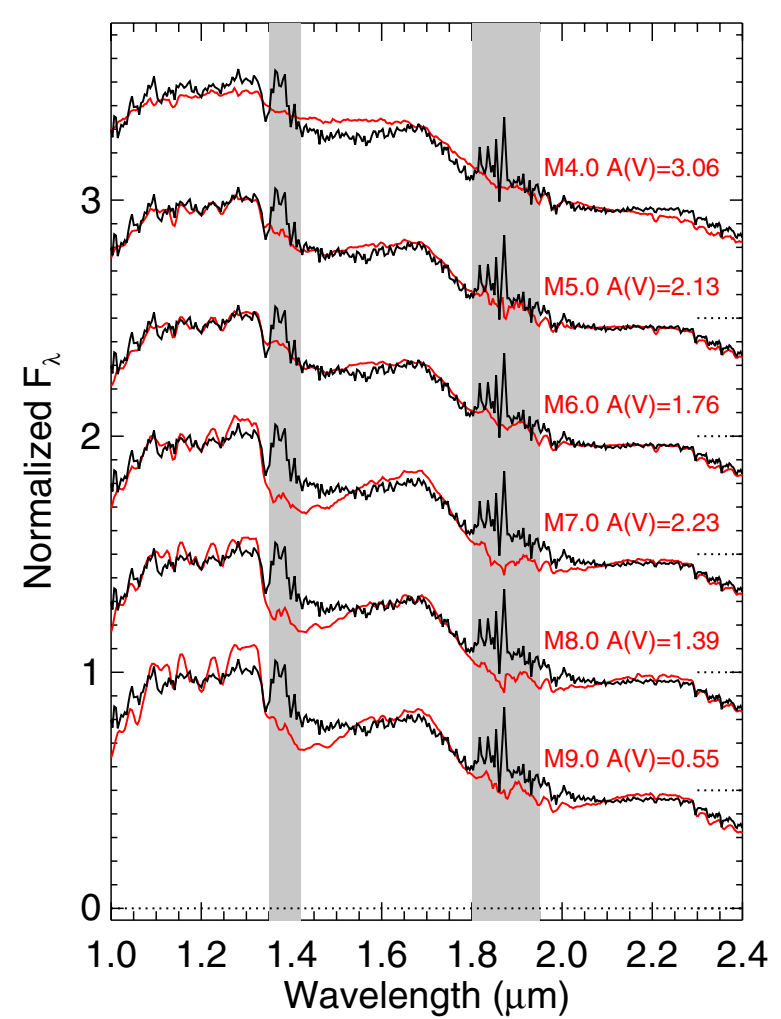

Figure 9. Comparison between the spectrum of V380 Ori B (black) and those of six M4-M9 dwarf field standard stars (red; Kirkpatrick et al. 1991; data from Burgasser et al. 2004, 2008) with different extinctions, showing good correspondence for M5-M6 stars with low extinction. The regions most affected by telluric absorption are shaded in gray, and were not used in the fits.

(A color version of this figure is available in the online journal.)

reddening law and $R_{V}=3.1$ (regions most affected by telluric absorption were not included). It is evident that the best fits occur around M5 and M6 with low values of extinction $A_{V} \sim 2$. V380 Ori is still intimately associated with its placental material, but given its high luminosity this association cannot last long, and so it probably is only a million years old or less. Assuming a similar age for V380 Ori B, a spectral type of M5.5 corresponds to the boundary between very low mass (VLM) stars and brown dwarfs (BDs). In Section 4 we discuss further the origin and evolutionary state of this borderline BD.

We note that Paschen- $\beta$ is in emission in V380 Ori B; emission in this line has been interpreted as evidence for magnetospheric accretion, or it may be evidence for a weak outflow from the object (Folha \& Emerson 2001; Whelan et al. 2004).

\subsection{HH 222 as a Giant Bow Shock}

The observations presented in Section 3 have made clear that HH 222 forms a giant bow shock moving straight away from V380 Ori. This, combined with the realization that the two infrared sources, IRS 1 and 2, at the center of HH 222 are likely giant elliptical galaxies, strongly suggests that there is no physical connection between HH 222 and the non-thermal radio continuum jet, but that they form an improbable line-ofsight association. This is fully consistent with the conclusions of Trejo \& Rodríguez (2010), who did not detect any proper motion of the radio jet.

Many, in fact most, giant HH flows are bipolar (e.g., Reipurth et al. 1997), so we have searched a deep Subaru image, identical to the one covering the $\mathrm{HH} 222$ field, but covering the region south of V380 Ori. Opposite to HH 222 we have found a new HH object, here called HH 1041, and seen in Figure 10. It is a large object, consisting of a main shock $\left(\sim 30^{\prime \prime}\right.$ wide) with additional fainter knots further east. Whereas the core of HH 222 is located at a P.A. of 331.2 relative to V380 Ori, then HH 1041 is at a P.A. of 151.6 . The difference is 179.6 , in other words the two shocks are precisely on opposite sides of V380 Ori, which strongly supports that they form a pair of shock and counter-shock from this star. While HH 222 and HH 1041 are symmetric in angle around V380 Ori, their distances to the star are not the same, HH 222 is 22 '.6 from V380 Ori, while $\mathrm{HH} 1041$ is only $17 \cdot 3$, which is $25 \%$ less. This, however, is often seen in giant $\mathrm{HH}$ flows, a prominent example is the nearby 


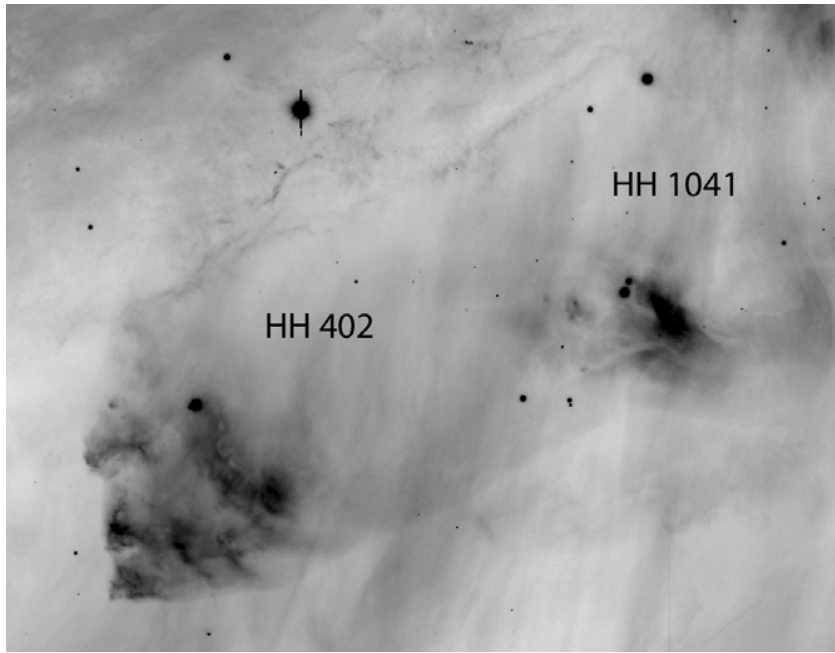

Figure 10. $\mathrm{H} \alpha$ image of the $\mathrm{HH} 402$ giant bow shock, with the new HH 1041 shock to the right. The image is about $5.8 \times 7.4$ arcmin, and north is up and east is to the left.

Table 3

Coordinates of Objects

\begin{tabular}{lccl}
\hline \hline Object & $\alpha_{2000}$ & $\delta_{2000}$ & \multicolumn{1}{c}{ Comments } \\
\hline HH 222 & $05: 35: 41.5$ & $-06: 23: 07$ & Center of "Streamers" \\
HH 222 & $05: 35: 42.0$ & $-06: 23: 01$ & "[S II]-bright knot" \\
HH 1031 & $05: 36: 06.4$ & $-06: 35: 33$ & \\
HH 35 & $05: 36: 22.4$ & $-06: 41: 50$ & Brightest knot \\
V380 Ori & $05: 36: 25.4$ & $-06: 42: 58$ & \\
V380 Ori B & $05: 36: 25.9$ & $-06: 43: 02$ & Faint companion at $\sim 9$ " \\
HH 130 & $05: 36: 45.7$ & $-06: 50: 07$ & Apex of bow shock \\
HH 1041 & $05: 36: 58.5$ & $-06: 58: 10$ & About 30" wide \\
\hline
\end{tabular}

HH 401/402 flow which is driven by the HH 1/2 source (Ogura 1995). HH 402 is located very close to HH 1041, and is also seen in Figure 10. Coordinates of the various objects are given in Table 3.

We have examined the path between V380 Ori and HH 222 in search of other shocked structures. Close to V380 Ori we see HH 35, originally discovered by Herbig (1974). Figure 11 shows a [S II] image of this object, which reveals a number of much fainter knots south of HH 35. Like HH 222, HH 35 lies northwest of V380 Ori, and the P.A. of the brightest knot in $\mathrm{HH} 35$ is $326^{\circ} 0$, within $5^{\circ}$ the same as that of $\mathrm{HH} 222$. The brightest knot in $\mathrm{HH} 35$ is also detected in $\mathrm{H}_{2} 2.12 \mu \mathrm{m}$ emission by Stanke et al. (2002), their knot SMZ 6-3, and they find another $\mathrm{H}_{2}$ emission knot, SMZ 6-8, almost symmetrically opposite V380 Ori, see their Figure 8. Other faint knots (HH 148) are found to the WSW of and very close to V380 Ori (Strom et al. 1986; Corcoran \& Ray 1995; Stanke et al. 2010), almost perpendicular to the HH 35/222 flow axis, but they are not visible in our images on account of the bright reflection nebula NGC 1999. They are, however, seen well in the [S II] HST image in Figure 6, which shows locations of the knots indicative of a gradually changing flow-axis. HH 148 is likely to represent a separate flow from one of the other components in the V380 Ori multiple system.

Further to the northwest, we have found a complex of faint HH knots, which we label HH 1031 (Figure 12). The brightest of these knots is located 8.8 northwest of V380 Ori, at a P.A. of $327.5,3.7$ less than that of $\mathrm{HH} 222$, but 1.5 more than that of $\mathrm{HH}$ 35. The smooth progression of separations and P.A.s of $\mathrm{HH}$

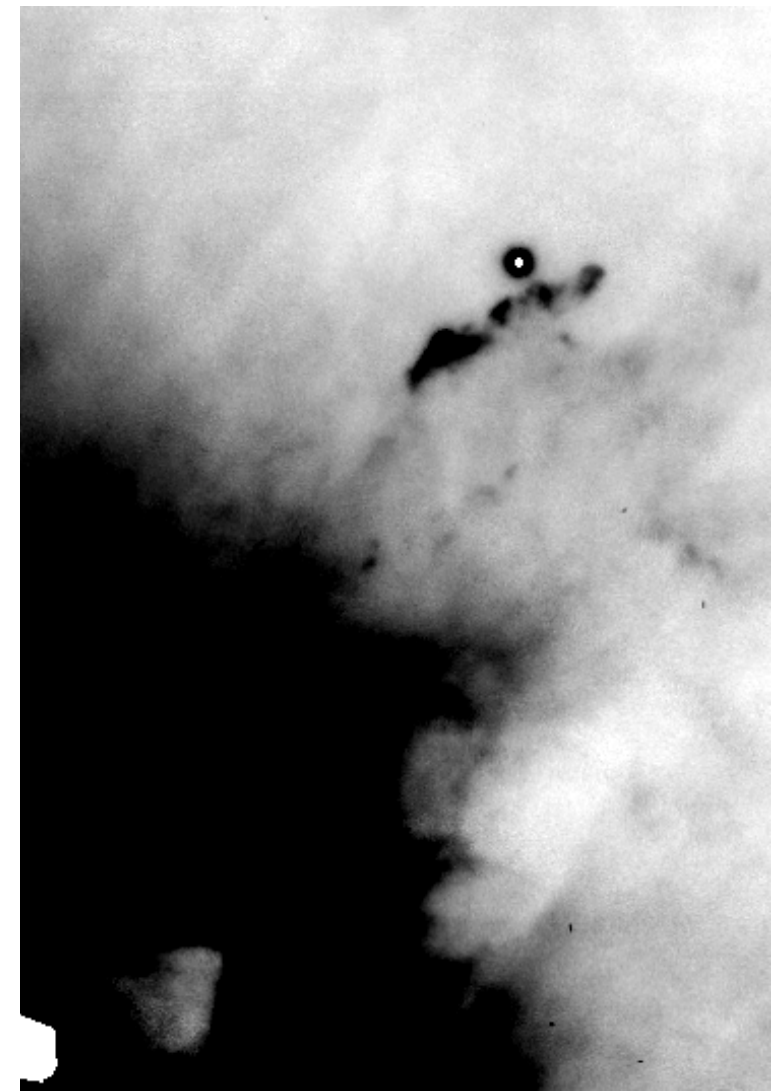

Figure 11. [S II] image of the HH 35 object associated with V380 Ori. The object is resolved into four main knots plus minor fainter shocks, and a group of small [S II] bright knots are associated with HH 35 to the south. Part of the saturated image of V380 Ori is seen to the lower left. The figure spans $75 \times$ 105 arcsec. North is up and east is to the left.

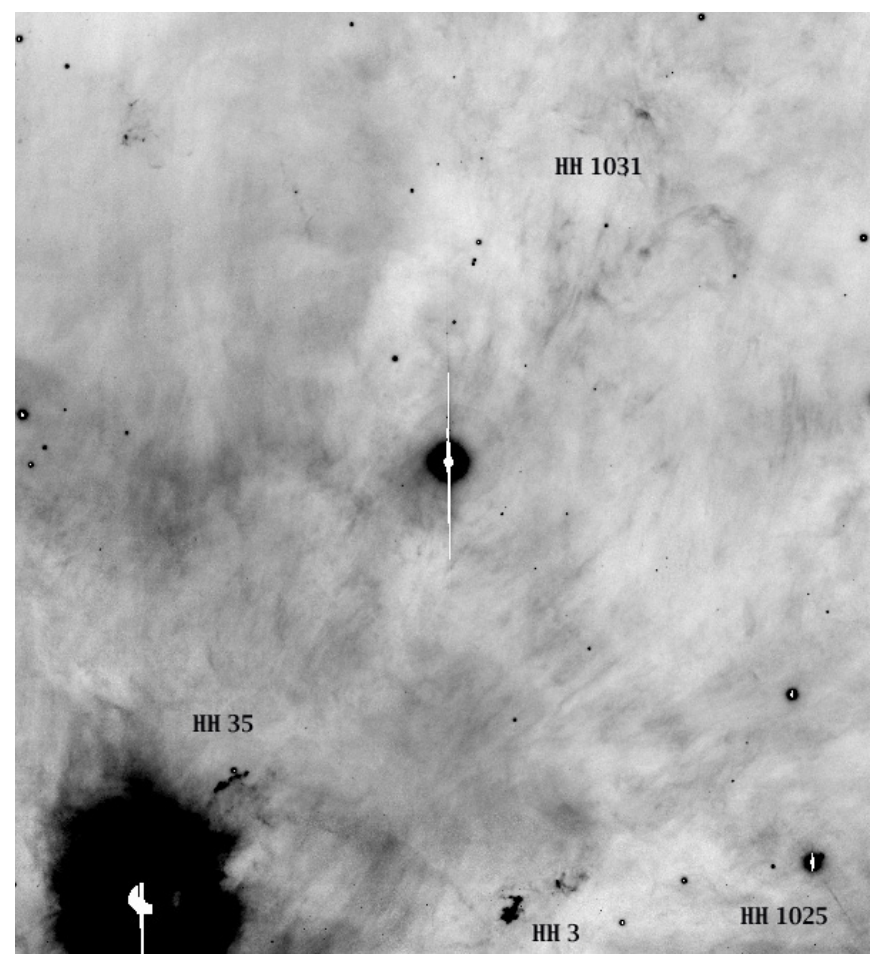

Figure 12. [S II] image of the HH 1031 region, taken at the Subaru telescope with SuprimeCam, with V380 Ori and HH 35 to the bottom left. North is up and east is to the left. 


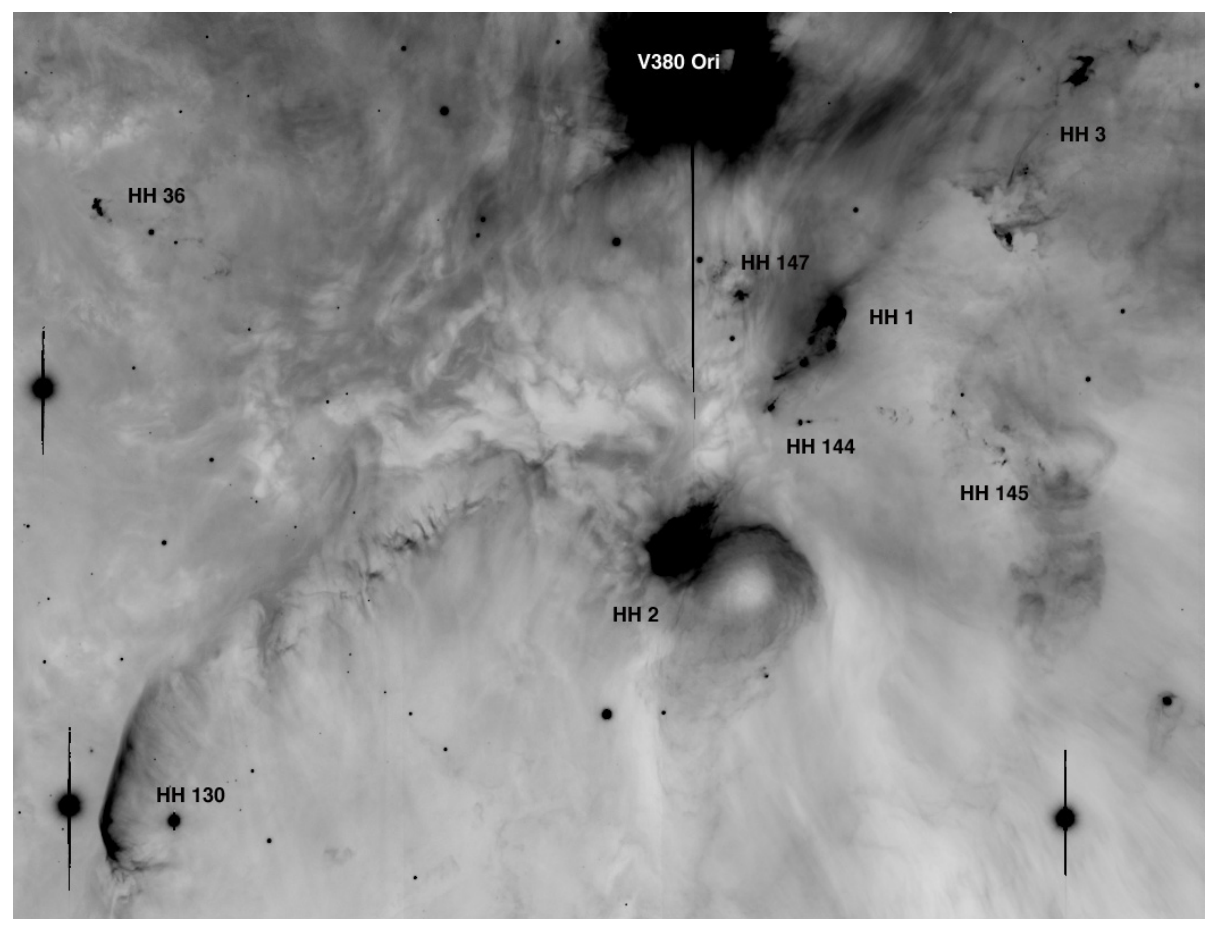

Figure 13. Widefield $\mathrm{H} \alpha$ panorama of the region south of V380 Ori, including the partial bow shock HH 130.

222, $\mathrm{HH}$ 1031, and HH 35 suggests that they all belong to the same main outflow, which has seen a gentle change in outflow angle.

If we look to the counterlobe, precisely 8.8 from V380 Ori at a P.A. of 144.9 we find the object HH 130, seen in Figure 13. This object was first noted by Reipurth (1985) and it was subsequently confirmed as an HH object by Ogura \& Walsh (1991) and Corcoran \& Ray (1995). Its morphology suggests that it is a partial bow shock interacting with the L1641 molecular cloud. The fact that $\mathrm{HH} 130$ is located at the precise same distance from V380 Ori as HH 1031 and that the two flows lie along the same line within a difference of only 2.6 strongly suggests that both represent simultaneous but opposite ejections from V380 Ori. The principal difference between the two objects is their brightness: $\mathrm{HH} 1031$ is a very faint $\mathrm{HH}$ object, whereas $\mathrm{HH} 130$ is a much brighter and larger object. This is probably related to the orientation of the V380 Ori outflow axis. HH 222 is slightly blueshifted (see Section 3.2), suggesting that the counterflow is slightly redshifted. Support for this comes from the millimeter observations of Morgan et al. (1991), who found a monopolar, redshifted, molecular outflow lobe associated with V380 Ori, located to its southeast (see also Corcoran \& Ray 1995, their Figure 19). Given that the L1641 cloud is behind V380 Ori, the southern slightly redshifted outflow lobe is more likely to interact with the molecular cloud than the northern lobe, creating a stronger shock. The region is complex, Stanke et al. (2000) found $\mathrm{H}_{2}$ shocks east and southeast of V380 Ori, which they ascribe to the giant L1641-N flow, and this might contribute to the molecular outflow.

Figure 14 provides an overview of the complete HH 222 outflow complex. The figure also shows the giant HH 401/402 flow associated with the HH 1/2 VLA source (Ogura 1995). The dimensions of the two giant flows are almost the same, and both show evidence for a second outflow episode (HH130/ 1031 for V380 Ori and HH 1/2 for the HH 1/2 VLA source), both of which are slightly oriented away from the principal flow axis.

\subsection{An Eruptive Event in the V380 Ori Quadruple System}

HH 222 is, together with the nearby HH 401 (see Figure 14), one of the largest and brightest giant $\mathrm{HH}$ bow shocks known. On the assumption that outflow activity and accretion is related, we may deduce that a particularly violent event occurred which created HH 222 and its counter shock HH 1041. What would trigger such an event in a young Herbig Ae/Be star? The key may be found in the multiple nature of V380 Ori.

When multiple systems are born they are likely to be nonhierarchical, that is, the time-averaged distances between components are comparable, and such configurations are inherently unstable and decay to a hierarchical configuration within typically a hundred crossing times (e.g., van Albada 1968; Valtonen \& Mikkola 1991). This transformation occurs as a result of a close triple encounter between components, during which energy and momentum can be transferred. Most such encounters occur during the protostellar stage (Reipurth 2000; Reipurth et al. 2010), which means that dynamical interactions occur not only between the stars, but even more so between their circumstellar disks. Such disk-disk interactions are particularly strong during a close triple encounter, leading to a massive accretion episode, and an ensuing powerful burst of mass loss. Such interactions in a newborn multiple system may appear as the well-known FUor eruptions (Bonnell \& Bastien 1992; Reipurth \& Aspin 2004).

We interpret the HH 222 complex within this scenario. Currently V380 Ori is a hierarchical quadruple system, with a close T Tauri companion in a 104 day orbit, a third body at a projected separation of $\sim 70 \mathrm{AU}$, and a VLM fourth body with a projected separation of $\sim 4000 \mathrm{AU}$. We posit that the system initially was non-hierarchical and decayed 28,000 yr ago or less, into a bound hierarchical multiple system, in the process creating the spectacular HH 222 shock structure we still today see traveling away from the star.

Reipurth \& Clarke (2001) suggested that ejection of stellar embryos from a dynamically evolving triple system could 


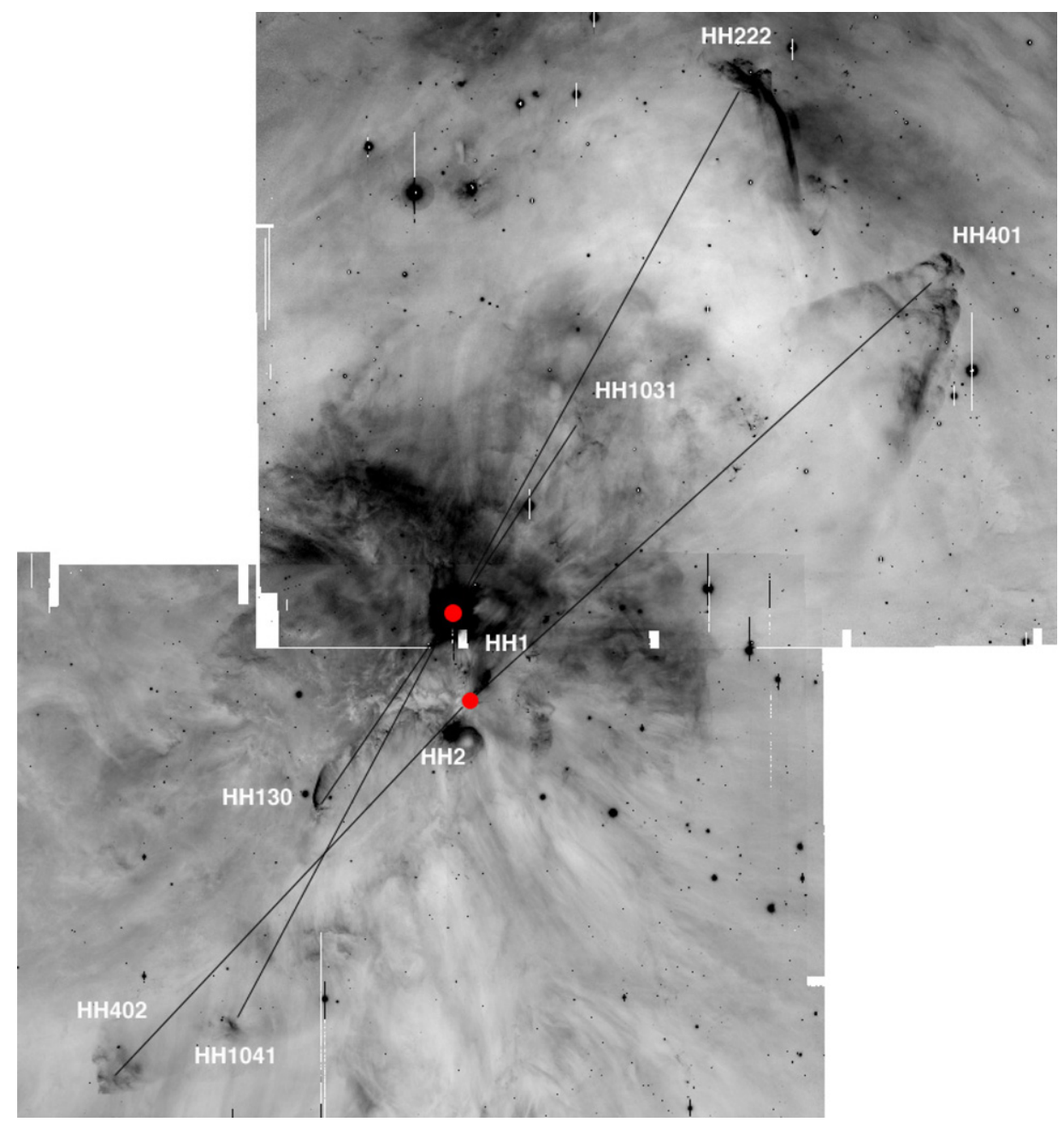

Figure 14. Giant flows in the V380 Ori and HH 1/2 region as seen on a composite of two H $\alpha$ images with SuprimeCam on the $8 \mathrm{~m}$ Subaru telescope. The driving sources, V380 Ori and HH 1/2 VLA are marked by red dots. Black lines show two almost straight flow lines.

(A color version of this figure is available in the online journal.)

explain the formation of at least some BDs. With a projected separation of only $\sim 4000$ AU from a compact triple system, the VLM/BD companion V380 Ori B appears likely to have had such an origin. The mean terminal velocity of such ejected bodies was found to be $1.13 \mathrm{~km} \mathrm{~s}^{-1}$ in the simulations presented by Reipurth et al. (2010). Assuming an ejection with an angle of $30^{\circ}$ from the plane of the sky, the most likely tangential velocity of $\mathrm{V} 380$ Ori $\mathrm{B}$ is then $0.98 \mathrm{~km} \mathrm{~s}^{-1}$. This suggests that V380 Ori B last time was close to V380 Ori and its other companions about 19,400 yr ago. Given the uncertainties involved in this estimate, this compares well with the upper limit of 28,000 yr derived from proper motions of HH 222 for the cataclysmic event that transformed the multiple system into its present dynamical configuration. If so, then V380 Ori B is at the moment most likely moving slowly away from V380 Ori. It may be escaping, or it may be in a bound orbit, in the latter case it is either in a stable or an unstable orbit. Reipurth \& Mikkola (2012) calculated the probabilities for being in any of these three states for different ages and projected separations of such small bodies; for an age of $1 \mathrm{Myr}$ and a projected separation of $4000 \mathrm{AU}$, the probability that V380 Ori B is escaping is low, only $\sim 10 \%$, whereas the probability of being in a stable bound orbit is $\sim 20 \%$, and in an unstable bound orbit $\sim 70 \%$. In other words, in all likelihood we are watching V380 Ori B in a bound but unstable orbit that during one of its future periastron passages will bring it into such close contact with the remaining three stars in the system that it will be ejected from the system and become a free-floating VLM or BD object.

In this interpretation, the second pair of $\mathrm{HH}$ objects, $\mathrm{HH} 1031$ and $\mathrm{HH} 130$, are due to a periastron passage by one of the other companions roughly 10,000 yr ago, and HH 35 represents the outflow activity due to a more recent periastron passage. These events, during which milder interactions took place, may be related to the orbital evolution of the two close companions of V380 Ori, which have evolved in a viscous medium that likely has shrunk their orbits to the present more compact configuration.

During periastron passages, the presence of another body near the central binary may apply a torque and cause precession of the jet engine, leading to a gradual shift of the outflow axis, as observed. This may also account for the presence of the faint $\mathrm{HH}$ objects HH 148 imaged by Corcoran \& Ray (1995) and lying very close to V380 Ori along an axis at a P.A. of approximately $250^{\circ}$ (see Figure 6); these faint $\mathrm{HH}$ objects are likely to originate from one of the two companions very close to V380 Ori itself.

\section{CONCLUSIONS}

We have carried out a detailed study of the strange HH 222 object, which coincides with a non-thermal radio jet toward the Orion clouds, and have obtained the following results. 
1. Measurements of the tangential velocity of HH 222 show a well-defined motion directly away from the nearby Herbig Be star V380 Ori.

2. Infrared images of the core of HH 222 show that the two previously identified sources located toward its core are likely giant elliptical galaxies belonging to a small cluster of galaxies, implying that there is no physical connection between the $\mathrm{HH}$ shock and the radio jet, but that they form an unexpected line-of-sight association. We are reminded that improbable does not mean impossible.

3. Spectroscopy and detailed imaging of the HH 222 bow shock reveals that its unusual morphology is due to a low density, limb-brightened sheet of shocked gas seen nearly edge on. Proper motions and radial velocities of $\mathrm{HH} 222$ show that it is slightly blueshifted, moving at an angle of only $12 \pm 8^{\circ}$ to the plane of the sky.

4. We find a bright and large $\mathrm{HH}$ object, $\mathrm{HH} 1041$, along the precise axis defined by $\mathrm{HH} 222$ and V380 Ori, but opposite to $\mathrm{HH} 222$. Whereas HH 222 is $3.0 \mathrm{pc}$ in projection from V380 Ori, HH 1041 is 2.3 pc away, leading to a total extent of $5.3 \mathrm{pc}$, making the HH 222 flow among the largest giant HH flows known.

5. Two other HH objects, the previously known HH 130 and a new faint object $\mathrm{HH}$ 1031, form a second pair located symmetrically around V380 Ori with almost the same flow angle as HH 222.

6. We find a young borderline BD $\sim 9^{\prime \prime}(\sim 4000$ AU in projection) from V380 Ori, making it a quadruple system. We interpret the above observations in terms of the dynamical evolution of an initially non-hierarchical multiple system, which has decayed into a bound hierarchical system, and during the initial close multiple encounter produced the giant HH 222/1041 bipolar HH flow, and at the same time ejected V380 Ori B into a wide orbit that is most likely unstable, eventually releasing the borderline BD to drift away.

We thank the referee, Thomas Stanke, for useful comments. B.R. thanks ESO for hospitality in Garching while much of this paper was written. This work is based in part on data collected at the Subaru Telescope, which is operated by the National Astronomical Observatory of Japan (NAOJ). I.A. would like to thank the staff of the ESO Paranal Observatory for the excellent support of the FORS team during the commissioning run (programme 60.A-9203(E). This project was supported by the Gemini Observatory, which is operated by the Association of Universities for Research in Astronomy, Inc., on behalf of the international Gemini partnership of Argentina, Australia, Brazil, Canada, Chile, the UK, and the US. M.S.C. and B.R. were Visiting Astronomers at the Infrared Telescope Facility, which is operated by the University of Hawaii under cooperative agreement No. NNX-08AE38A with the National Aeronautics and Space Administration, Science Mission Directorate, Planetary Astronomy Program. This research has made use of the SIMBAD database, operated at CDS, Strasbourg, France, and of NASA's Astrophysics Data System Bibliographic Services. B.R. acknowledges support by the National Science Foundation under grant NSF AST-0407005. This material is based upon work supported by the National Aeronautics and Space Administration through the NASA Astrobiology Institute under cooperative agreement No. NNA09DA77A issued through the
Office of Space Science. This research has benefited from the SpeX Prism Spectral Libraries, maintained by Adam Burgasser at http://www.browndwarfs.org/spexprism.

\section{REFERENCES}

Alecian, E., Wade, G. A., Catala, C., et al. 2009, MNRAS, 400, 354

Bonnell, I., \& Bastien, P. 1992, ApJL, 401, L31

Burgasser, A. J., Liu, M. C., Ireland, M. J., Cruz, K. L., \& Dupuy, T. J. 2008, ApJ, 681,579

Burgasser, A. J., McElwain, M. W., Kirkpatrick, J. D., et al. 2004, AJ, 127, 2856

Cardelli, J. A., \& Böhm, K. H. 1984, ApJ, 285, 613

Cardelli, J. A., Clayton, G. C., \& Mathis, J. S. 1989, ApJ, 345, 245

Castets, A., Reipurth, B., \& Loinard, L. 2004, A\&A, 427, 895

Cohen, M., \& Schwartz, R. D. 1983, ApJ, 265, 877

Corcoran, D., \& Ray, T. P. 1995, A\&A, 301, 729

Crusius-Wätzel, A. R. 1990, ApJL, 361, L49

Curiel, S., Rodríguez, L. F., Moran, J. M., \& Cantó, J. 1993, ApJ, 415, 191

Devine, D., Bally, J., Reipurth, B., \& Heathcote, S. 1997, AJ, 114, 2095

Folha, D. F. M., \& Emerson, J. P. 2001, A\&A, 365, 90

Hamann, F., \& Persson, S. E. 1992, ApJS, 82, 285

Henriksen, R. N., Ptuskin, V. S., \& Mirabel, I. F. 1991, A\&A, 248, 221

Herbig, G. H. 1946, PASP, 58, 163

Herbig, G. H. 1960, ApJS, 4, 337

Herbig, G. H. 1974, LicOB, 658, 1

Heyer, M. H., Morgan, J., Schloerb, F. P., Snell, R. L., \& Goldsmith, P. F. 1992, ApJL, 395, L99

Hodapp, K.-W. 1994, ApJS, 94, 615

Kirkpatrick, J. D., Henry, T. J., \& McCarthy, D. W. 1991, ApJS, 77, 417

Leinert, C., Richichi, A., \& Haas, M. 1997, A\&A, 318, 472

Menten, K. M., Reid, M. J., Forbrich, J., \& Brunthaler, A. 2007, A\&A, 474, 515

Millan-Gabet, R., Schloerb, F. P., \& Traub, W. A. 2001, ApJ, 546, 358

Morgan, J. A., Snell, R. L., \& Strom, K. M. 1990, ApJ, 362, 274

Morgan, J. A., Schloerb, F. P., Snell, R., \& Bally, J. 1991, ApJ, 376, 618

Morgan, W. W., \& Sharpless, S. 1946, ApJ, 103, 249

Nakamura, F., Kamada, Y., Kamazaki, T., et al. 2012, ApJ, 750, A174

Ogura, K. 1995, ApJL, 450, L23

Ogura, K., \& Walsh, J. R. 1991, AJ, 101, 185

Raga, A. C., Curiel, S., Rodríguez, L. F., \& Cantó, J. 2000, A\&A, 364, 763

Rayner, J. T., Toomey, D. W., Onaka, P. M., et al. 2003, PASP, 115, 362

Reipurth, B. 1985, A\&AS, 61, 319

Reipurth, B. 1999, A General Catalogue of Herbig-Haro Objects, 2nd ed., http://casa.colorado.edu/hhcat

Reipurth, B. 2000, AJ, 120, 3177

Reipurth, B., \& Aspin, C. 2004, ApJL, 608, L65

Reipurth, B., Bally, J., \& Devine, D. 1997, AJ, 114, 2708

Reipurth, B., Chini, R., Krügel, E., Kreysa, E., \& Sievers, A. 1993, A\&A, 273,221

Reipurth, B., \& Clarke, C. 2001, AJ, 122, 432

Reipurth, B., \& Mikkola, S. 2012, Natur, 492, 221

Reipurth, B., Mikkola, S., Connelley, M., \& Valtonen, M. 2010, ApJL, 725, L56 Reipurth, B., \& Sandell, G. 1985, A\&A, 150, 307

Rodríguez, L. F., Curiel, S., Moran, J. M., et al. 1989, ApJL, 346, L85

Rossi, C., Errico, L., Friedjung, M., et al. 1999, A\&AS, 136, 95

Smith, K. W., Balega, Y. Y., Duschl, W. J., et al. 2005, A\&A, 431, 307

Solf, J., Böhm, K.-H., \& Raga, A. C. 1988, ApJ, 334, 229

Stanke, T., McCaughrean, M. J., \& Zinnecker, H. 2000, A\&A, 355, 639

Stanke, T., McCaughrean, M. J., \& Zinnecker, H. 2002, A\&A, 392, 239

Stanke, T., Stutz, A. M., Tobin, J. J., et al. 2010, A\&A, 518, L94

Stelzer, B., Micela, G., Hamaguchi, K., \& Schmitt, J. H. M. M. 2006, A\&A, 457, 223

Strom, K. M., Strom, S. E., Wolff, S. C., Morgan, J., \& Wenz, M. 1986, ApJS, 62,39

Trejo, A., \& Rodríguez, L. F. 2010, RMxAA, 46, 357

Valtonen, M., \& Mikkola, S. 1991, ARA\&A, 29, 9

van Albada, T. S. 1968, BAN, 19, 479

Warren-Smith, R. F. 1983, MNRAS, 205, 349

Whelan, E. T., Ray, T. P., \& Davis, C. J. 2004, A\&A, 417, 247

Wilson, B. A., Dame, T. M., Masheder, M. R. W., \& Thaddeus, P. 2005, A\&A, 430, 523

Yusef-Zadeh, F., Cornwell, T. J., Reipurth, B., \& Roth, M. 1990, ApJL, 348, L61 